\title{
Risk Factors Analysis of Acute Kidney Injury Incidence Following Coronary Artery Bypass Graft with Cardiopulmonary Bypass in Indonesia
}

\author{
Ghuraba Adisurya, Kun Arifi Abbas*, Philia Setiawan, Fajar Perdhana
}

\author{
Anesthesiology and Intensive Care Department, Faculty of Medicine \\ Universitas Airlangga Surabaya, 60286, Indonesia
}

\section{*Corresponding author details: Kun Arifi Abbas; kunarifiunair@gmail.com}

\begin{abstract} $\mathrm{CPB}$ and also lower plasma osmolarity

\section{INTRODUCTION}

The incidence of Acute Kidney Injury (AKI) after heart surgery, whether using Cardiopulmonary Bypass (CPB) or not, is now known to reach $49.9 \%$ (Prabhu et al., 2010). The medical records in one of the hospital in Indonesia showed that the incidence of AKI after CPB in 2019 reached $69.8 \%$.
\end{abstract}

Introduction: The incidence of postoperative CABG AKI in Indonesia, one of which is in the city of Surabaya, is known to reach $69.8 \%$. For this reason, this study was prepared with the aim of analyzing the risk factors of Acute Kidney Injury Incidence following coronary artery bypass graft with cardiopulmonary bypass.

Method: The study conducted a prospective analytic study on CABG patients with the CPB technique without a history of previous kidney disease at a hospital in Indonesia in the period 2020.

Results: Through the results of the study, it was found that 14 people were diagnosed as AKI after surgery, of which 11 were men. On the other hand, it is also known that AKI patients also experience a longer duration of

Conclusion: Of all the factors it is known that eGFR is an independent risk factor for AKI.

Keywords: acute Kidney Injury; coronary artery bypass graft; cardiopulmonary bypass

Coronary Artery Bypass Graft (CABG) is a procedure to replace coronary arteries that have atherosclerotic plaque occlusion with arteries or veins from other parts of the body, so that blood flow to the heart muscle returns to normal (Alexander \& Smith, 2016). his procedure is the most common thoracic and cardiovascular surgical procedure performed by medical professionals (Squiers \& Mack, 2018). In one hospital in Indonesia, namely in Surabaya, it is known that CABG procedures in 2018 and 2019 were recorded at 82 and 64 times, respectively.

There are several conditions associated with the incidence of AKI, namely old age and female gender, preoperative cardiac dysfunction, emergency surgery, peripheral arterial disease, reinvention, diabetes requiring insulin, intraoperative aprotinin use, patients with obstructive pulmonary disease. chronic obstructive pulmonary disease (COPD), and preoperative renal dysfunction (Shin et al., 2016), (Vives et al., 2019), (Yi et al., 2016). A study showed that optimal blood pressure during perioperative will influence the occurrence of AKI. On the one hand, the duration of CPB is also a very important factor in this problem.

Investigating further, it is also known that plasma osmolarity is the most important factor that determines the direction of fluid movement in the body. The relationship between plasma osmolarity and the incidence of postoperative AKI is still very rarely studied.
Glomerular filtration rate (GFR) is an important component that determines the occurrence of AKI in post cardiovascular patients. Research has shown that GFR $<30$ before surgery is a risk factor for postoperative AKI (Silva et al., 2018). his is consistent with other studies which state that preoperative GFR can be used to predict the risk of postoperative AKI (Wu et al., 2019). Therefore, this study was structured to analyze the risk factors of Acute Kidney Injury Incidence following coronary artery bypass graft with cardiopulmonary bypass in Indonesia.

\section{METHOD}

This research is an analytic observational study which aims to determine the causal relationship between variables. This research was conducted in the Central Surgical Installation, nursing ward, and ICU RSUD Dr. Soetomo, Surabaya City, Indonesia at the end of 2020. The target of this study was all patients who underwent CABG surgery with the CPB technique at RSUD Dr. Soetomo Surabaya.

\section{RESULT}

\section{Research Characteristics}

This research was conducted in RSUD Dr. Soetomo Surabaya Indonesia. It is known that the inclusion criteria of this study selected by the researcher were all patients who underwent CPB with complete examination data. The sample in this study was dominated by men with a total of $17(81.0 \%)$ study samples with uncontrolled comorbidities with a total of $18(85.7 \%)$ study samples. The majority of patients undergoing CPB had AKI with a total of 13 (61.9\%) study samples.

Hemodynamic Analysis (MAP) on the incidence of AKI Post Cardiopulmonary Bypass

The results of this study showed that patients who did not have AKI had a lower MAP than those who did not experience 
both on pre-induction ( $99.5 \pm 13.0$ vs $97.8 \pm 12.3)$ and durante $(82.2 \pm 15.1$ vs $79.9 \pm 12.6)$.
The difference between MAP duration of surgery ( $p$-value 0.681 ) and preinduction MAP ( $p$-value 0.771 ) was not significantly related based on statistical calculations.

TABLE 1: Differences in MAP based on the incidence of AKI.

\begin{tabular}{|c|c|c|c|}
\hline MAP & n & Average \pm Standard Deviation & P Value \\
\hline \multicolumn{4}{|l|}{ Pre CPB } \\
\hline AKI & 14 & $99,11 \pm 16,06$ & \multirow{2}{*}{0,257} \\
\hline Without AKI & 7 & $91,57 \pm 7,55$ & \\
\hline \multicolumn{4}{|l|}{ Low CPB Durante } \\
\hline AKI & 14 & $44,79 \pm 11,53$ & \multirow{2}{*}{0,415} \\
\hline Without AKI & 7 & $40,57 \pm 9,48$ & \\
\hline \multicolumn{4}{|l|}{ High CPB Durante } \\
\hline AKI & 14 & $75,33 \pm 10,88$ & \multirow{2}{*}{0,368} \\
\hline Without AKI & 7 & $70,00 \pm 15,39$ & \\
\hline \multicolumn{4}{|l|}{ Low Early ICU } \\
\hline AKI & 14 & $69,93 \pm 15,50$ & \multirow{2}{*}{0,735} \\
\hline Without AKI & 7 & $72,29 \pm 13,34$ & \\
\hline \multicolumn{4}{|l|}{ High Early ICU } \\
\hline AKI & 14 & $90,00 \pm 14,26$ & \multirow{2}{*}{0,760} \\
\hline Without AKI & 7 & $92,29 \pm 19,09$ & \\
\hline
\end{tabular}

The test results showed that there was no significant difference in MAP Pre CPB, low/high Durante CPB and low/high early ICU between the AKI and no AKI groups $(\mathrm{p}>$ 0.05).
Analysis of Cardiopulmonary Bypass Time on the Incidence of AKI Post Cardiopulmonary Bypass

Table 2 below shows the analysis of the differences between cardiopulmonary bypass time and the incidence of AKI in patients after cardiopulmonary bypass.

TABLE 2: The difference between CBP time and the incidence of AKI.

\begin{tabular}{|c|c|c|c|}
\hline \multirow{2}{*}{ CPB Time } & \multicolumn{3}{|c|}{ AKI Incidence (Mean \pm SD) } \\
\cline { 2 - 4 } & Yes & No & p-value \\
\cline { 2 - 4 } & $139,1 \pm 64,0$ & $102,8 \pm 18,0$ & $0,179^{*}$ \\
\hline
\end{tabular}

Table 2 shows that patients with AKI had a higher CBP time than those without AKI (139.1 \pm 64.0 vs $102.8 \pm 18.0)$, but the difference was not statistically significant ( $\mathrm{p}$-value $0.179)$.
Osmolarity Analysis with AKI Post Cardiopulmonary Bypass Incidence

Table 2 below shows the difference between the calculation of osmolarity and the incidence of AKI in patients who have undergone CPB.

TABLE 3: The difference between osmolarity and the incidence of AKI.

\begin{tabular}{|c|c|c|c|}
\hline \multirow{2}{*}{ Osmolarity } & \multicolumn{3}{|c|}{ AKI Incidence (Mean \pm SD) } \\
\cline { 2 - 4 } & Yes & No & p-value \\
\cline { 2 - 4 } & $315,2 \pm 60,7$ & $339,8 \pm 27,4$ & $0,296^{*}$ \\
\hline
\end{tabular}

Table 3 shows that patients without AKI had a higher osmolarity than those with AKI $(339.8 \pm 27.4$ vs $315.2 \pm$ 60.7). This comparison is not significant based on statistical calculations (p-value 0.296).

The results of the free sample T test and the Mann Whitney test showed that there was no significant difference in the Osmolarity of Pre CPB, Durante CPB and early ICU between the AKI and without AKI groups ( $p>0.05)$.

\section{Estimated-GFR Analysis of AKI Post Cardiopulmonary Bypass}

The data shows that patients with AKI had a lower e-GFR than those without AKI $(51.1 \pm 15.8$ vs $76.3 \pm 20.1)$.
The difference was significant based on statistical calculations (p-value 0.005).

The results of the free sample T test and the Mann Whitney test showed that there was no significant difference in the eGFR of Pre CPB and Durante CPB between the AKI and no AKI groups $(p>0.05)$, and there was a significant difference in the early ICU eGFR between the AKI and without AKI groups $(\mathrm{p}<0.05)$.

Analysis of Other Risk Factors for Post-CPB AKI Incidence Table 3 below shows the difference between serum albumin levels and the incidence of AKI in patients who have undergone CPB. 
TABLE 4: The difference between serum alumina levels and the incidence of AKI.

\begin{tabular}{|l|c|c|c|}
\cline { 2 - 4 } \multicolumn{1}{c|}{} & \multicolumn{3}{c|}{ AKI Incidence (Mean \pm SD) } \\
\cline { 2 - 4 } \multicolumn{1}{c|}{} & Yes & No & p-value \\
\hline Preinduction Albumin & $3,65 \pm 0,14$ & $3,64 \pm 0,21$ & $0,894^{+}$ \\
\hline Durante Albumin & $2,47 \pm 0,33$ & $2,50 \pm 0,32$ & $0,855^{+}$ \\
\hline Albumin Early ICU & $3,27 \pm 0,22$ & $3,25 \pm 0,19$ & $0,823^{+}$ \\
\hline
\end{tabular}

Table 4 shows that patients with AKI had higher albumin levels than those without AKI during preinduction (3.65 \pm 0.14 vs. $3.64 \pm 0.21$, p-value 0.894$)$ and early ICU ( $3.27 \pm$ 0.22 vs. $3.25 \pm 0.19$, p-value 0.823 , while on Durante the opposite result was obtained $(2.47 \pm 0.33$ vs. $2.50 \pm 0.32$, $\mathrm{p}$-value 0.855 ). This comparison is not significant based on statistical calculations.

\section{Analysis of Serum Delta Albumin on the Incidence of Post-CPB an AKI}

This study shows that patients with AKI had higher albumin delta than those without AKI during durantepreinduction $(-1.18 \pm 0.35$ vs. $-1.14 \pm 0.27$, p-value 0.597$)$, whereas the opposite result was obtained in early ICUpreinduction $(-0.37 \pm 0.21$ vs $-0.38 \pm 0.13$, p-value 0.907$)$ and early ICU-durante ( $-0.37 \pm 0.21$ vs. -0$)$. $38 \pm 0.13$, pvalue 0.907$)$. This comparison is not significant based on statistical calculations.

\section{Analysis of Vasoactive Inotropic Score (VIS) on the Incidence of Post-CPB}

The data shows that patients with AKI had a higher vasoactive inotropic score (VIS) than those without AKI during the duration of the period (89.6 \pm 277 vs. 0 , p-value 0.198$)$ and early ICU ( $19.5 \pm 62$ vs. $4.6 \pm 5$, p-value 0.755$)$. This comparison is not significant based on statistical calculations.

\section{Analysis of Delta Vasoactive Inotropic Score (VIS) on the Incidence of AKI Post-CPB}

The study shows that patients with AKI had a higher delta vasoactive inotropic score (VIS) than those without AKI during durante-preinduction $(89.6 \pm 277$ vs 0 , p-value $0.198)$ and early ICU-preinduction (19. , $5 \pm 62$ vs. $4.6 \pm 5$, p-value 0.755 , whereas in early ICU-durante the opposite result was obtained ( $-70.0 \pm 216$ vs. $4.6 \pm 5$, p-value 0.286$)$. This comparison is not significant based on statistical calculations.

\section{Hemoglobin Analysis of Post-CPB AKI Incidence}

The data shows that patients with AKI had preoperative hemoglobin levels $(12.90 \pm 1.50$ vs. $14.04 \pm 1.49$, p-value $0.115)$, hemoglobin durante CPB $(8.55 \pm 1.36$ vs $9.17 \pm 1.33$, p-value 0.332 ) and early ICU hemoglobin ( $10.26 \pm 1.75$ vs. $10.86 \pm 1.13$, p-value 0.428 ) was lower than in patients without AKI, but not significant. Meanwhile, pre-CPB hemoglobin levels were lower in patients without AKI than those with AKI, ie $12.01 \pm 3.34$ vs $12.71 \pm 1.34$, p-value 0.614 , but not significant. Then, this study shows that patients with AKI received aortic crossclamp treatment for a longer time than those without AKI, but this value was not statistically significant ( $84.93 \pm 34.50$ minutes vs. $64.71 \pm 16.50$ minutes, p-value 0.145 ).

\section{DISCUSSION}

Acute kidney injury (AKI) is a disease that covers a wide spectrum of kidney damage and often causes acute kidney failure (ARF). It is known that AKI usually causes an increase in serum creatinine ( $\mathrm{SCr}$ ) of more than $50 \%$ (Kasper; Denis L; et al, 2018).

Acute kidney injury can be associated with a person's metabolic condition depending on the severity and duration of the disease (Antonucci E et al., 2014; Kidney Disease: Improving Global Outcomes (KDIGO) CKD Work Group, 2013). A systematic review found that AKI occurs in one in five adults and one in three children hospitalized with acute illness. There are even studies which state that the incidence of AKI is most common in elderly, male and black patients (Rewa 0 \& Bagshaw SM, 2014).

The results of this study showed that the mean age \pm SD of the patients in this study was $58.7 \pm 8.5$ years. A variety of patient-specific factors and contexts can alter the risk of AKI. Old and very young patients are particularly susceptible to this disorder. Although older age has consistently been shown to increase the risk of AKI. However, it is known that elderly patients with AKI are much less likely to receive renal replacement therapy than younger patients (Rewa 0 \& Bagshaw SM, 2014).

Tracing deeper, it is known that chronic kidney disease is one of the factors in the risk of death. This can be evidenced by its integration into various clinical practice guidelines and risk prediction scores for the development of postprocedural AKI and the need for renal replacement therapy (Aru W; Idrus A; Marcelus S; et al, 2013; Rewa O \& Bagshaw SM, 2014). On the other hand, other diseases that can increase the risk of AKI are non-renal comorbidities such as diabetes, hypertension, vascular disease, and chronic obstructive pulmonary disease which apparently can be an important risk in the process of developing AKI (Aru W; Idrus A; Marcelus S; et al, 2013; Rewa O \& Bagshaw SM, 2014).

The Relationship between Hemodynamics and AKI Incidence in Post CPB Patients

This study showed that patients who did not have AKI had a lower MAP than those who had AKI both at pre-induction $(97.8 \pm 12.3$ vs. $99.5 \pm 13.0)$ and durante $(79.9 \pm 12.6)$. vs $82.2 \pm 15.1)$. The difference between MAP duration of surgery ( $p$-value 0.681 ) and preinduction MAP ( $p$-value 0.771 ) was not significantly related based on statistical calculations.

A study analyzing a subgroup of 127 patients found that patients with AKI had a lower mean MAP time than those without AKI. Thus, the investigators concluded that a MAP of 72-82 $\mathrm{mmHg}$ may be necessary to prevent AKI in patients with septic shock (Badin J et al., 2011). Blood pressure is associated with the need for renal replacement therapy, maximal creatinine concentration, and urine output. MAP $<75 \mathrm{mmHg}$ predicts the need for renal replacement therapy (Dunser MW et al., 2009). Hemodynamic factors are important in determining renal oxygenation because renal oxygen delivery is proportional to renal blood flow (Evans RG et al., 2013).

This mechanism is adaptive because it facilitates the balance of intake with oxygen demand. However, the renal circulation appears to be relatively insensitive to the local effects of hypoxia and hyperoxia. This is because blood flow is generally decreased during arterial hypoxemia caused by nerve activation of the chemoreceptors. Thus, renal blood flow is also decreased in humans during hypoxemia (Evans RG et al., 2013). 
An increase in renal blood flow has been observed in conscious rats sedated during the hypoxaemic process. Through this process, it is known that kidney function is not hindered by these changes. Instead, it was found that there was no strong response from the renal blood vessels (Evans RG et al., 2013).

An increase in renal blood flow has been observed in conscious rats sedated during the hypoxaemic process. Through this process, it is known that kidney function is not hindered by these changes. Instead, it was found that there was no strong response from the renal blood vessels (Evans RG et al., 2013).

\section{The Relationship between CBP Time and AKI Incidence in CPB Patients}

This study also showed that patients with AKI had higher CBP times than those without AKI $(139.1 \pm 64.0$ vs $102.8 \pm$ 18.0). There are few studies on the risk of postoperative AKI in patients undergoing cardiac surgery. Under these conditions they did not include the $\mathrm{CPB}$ time in the multivariate logistic regression equation. As well as a study conducted, which reported that CPB timing did not affect the development of postoperative AKI. In fact, these results became controversial enough to be associated with patient cohort confounders (Kowalik MM et al., 2011).

These results are consistent with a study in 851 elective thoracic aortic surgery patients, in which they found a longer CPB time (per $10 \mathrm{~min}$ ) to be a significant risk factor for postoperative AKI (OR = 1.09, 95\% CI: 1, 05-1.12; $\mathrm{P}=$ 0.001) (Kumar ABMF et al., 2014). On the other hand, there was a study showing 586 patients who underwent elective hemiarchal aortic reconstruction with CPB time ( $\mathrm{min}$ ) (OR $=1.01,95 \%$ CI: 1.00-1.01; P = 0.03). In addition, several other studies have found that CPB time is an independent predictor of postoperative AKI in patients undergoing cardiac and vascular surgery (Arnaoutakis GJ et al., 2016).

The potential mechanism linking the timing of CPB and AKI is unclear. A study examined the association between hemolysis and postoperative AKI in approximately 42 children undergoing cardiac surgery with CPB. Through this study they found that significant hemolysis occurred which could be associated with the postoperative development of AKI (Mamikonian LS et al., 2014).

Reducing CPB-induced hemolysis or reducing the effects of CPB-induced hemolysis by augmenting existing endogenous mechanisms for lowering free hemoglobin levels could be ways to more quickly treat excess plasma free hemoglobin, reduce oxidant injury, and minimize acute toxic effects. hemolysis and reduce the incidence of AKI (Mamikonian LS et al., 2014).

\section{Relationship between Osmolarity and AKI Incidence in CPB Patients}

Osmolality can be defined as the number of osmoles of solute per liter (solvent \pm solute), so it is expressed in milliosmoles per liter. Osmolarity is the concentration of solute per kilogram of solvent, and has units of milliosmoles per kilogram. It is the preferred unit in clinical practice because it is strong against plasma fat and protein content, whereas osmolarity is not. Extracellular osmolarity in humans is generally $290 \pm 10 \mathrm{mOsm} / \mathrm{kg}$ (Butterworth JF; et al, 2018; Guyton AC; Hall JE, 2016).

Through this study, it was found that patients who did not have AKI had a higher osmolarity than those who had AKI $(315.2 \pm 60.7$ vs $315.2 \pm 60.7)$, although this comparison was not significant based on statistical calculations (pvalue 0.296 ). The incidence of AKI was highest in those with the highest osmolarity and the lowest in the intermediate osmolarity.
High osmolarity independently predicted the development of AKI (OR 2.00, 95\% CI 1.26-3.19, p = 0.003). Patients with the highest osmolarity had a higher 1-year risk of death than patients with the lowest osmolarity (HR 2.11, 95\% CI 1.10$4.15 ; \mathrm{p}=0.031)$. Osmolarity is a valid and easily obtained predictor of AKI after PCI (Farhan S et al., 2019).

\section{Relationship between Estimated GFR and AKI Incidence in CPB Patients}

In this study, patients with AKI had a lower e-GFR than those without AKI ( $51.1 \pm 15.8$ vs $76.3 \pm 20.1)$. The difference was significant based on statistical calculations (p-value 0.005). Another study showed that preoperative renal dysfunction (eGFR $<60 \mathrm{~mL} / \mathrm{min}$, creatinine $>2.1 \mathrm{mg} / \mathrm{dL}$ ) was associated with AKI after cardiac surgery(Vives $M$ et al., 2019). Preoperative renal dysfunction risk factors are the most predictive factors in predicting the incidence of AKI after cardiac surgery.

\section{Analysis of the Relationship between Other Risk Factors on the Incidence of AKI in Post CPB Patients}

Patients with AKI had higher albumin levels than those without AKI during preinduction $(3.65 \pm 0.14$ vs. $3.64 \pm$ 0.21$, p-value 0.894$)$ and early ICU $(3.27 \pm 0,22$ vs $3.25 \pm$ 0.19 , p-value 0.823 , while in Durante it was not $(2.47 \pm$ 0.33 vs. $2.50 \pm 0.32$, p-value 0.855 ). This comparison is not significant based on statistical calculations. Patients with AKI had higher albumin delta than those without AKI during the duration of preinduction $(-1.18 \pm 0.35$ vs. -1.14 \pm 0.27 , p-value 0.597), whereas in the early ICUpreinduction $(-0.37 \pm 0.21$ vs $-0.38 \pm 0.13$, p-value 0.907$)$ and early ICU-durante $(-0.37 \pm 0.21$ vs $-0.38 \pm 0.13$, p value 0.907$)$. This comparison is not significant based on statistical calculations.

This study showed that serum albumin levels before surgery were higher after surgery. This is similar to another study which showed that albumin levels after cardiac surgery were significantly lower than before surgery and the proportion of patients with hypoalbuminemia increased from $9.6 \%$ to $27.6 \%$. The incidence of AKI in the hypoalbuminemia group was significantly higher than in the non-hypoalbuminemic group and the severity of AKI in the hypoalbuminemic group was increased compared to the non-hypoalbuminemic group. This condition can lead to prolonged hospitalization (Shufang L et al., 2020).

On the other hand, the vasoactive-inotropic score (VIS) is calculated as the sum of the requirements of all inotropics and vasoconstrictors administered, thus reflecting the need for pharmacological support of the cardiovascular system. Vasoactive-inotropic score is one indicator that can show increased morbidity and mortality, after surgery and heart attacks (Butterworth JF; et al, 2018; Koponen T et al., 2018).

Meanwhile, in this study, it was also found that patients with AKI had preoperative hemoglobin levels $(12.90 \pm 1.50$ vs $14.04 \pm 1.49$, p-value 0.115$)$, hemoglobin durante CPB (8.55 \pm 1.36 vs $9.17 \pm 1.33$, p-value 0.332 ). and early ICU hemoglobin ( $10.26 \pm 1.75$ vs $10.86 \pm 1.13$, p-value 0.428$)$ was lower than in patients without AKI, but not significant. While pre-CPB hemoglobin levels were lower in patients without AKI than those with AKI, namely $12.01 \pm 3.34$ vs $12.71 \pm 1.34$, p-value 0.614 , but not significant.

There was an association between the combination of 3 risk factors (preoperative anemia, intraoperative anemia, and red blood transfusion) on the day of $\mathrm{CPB}$ and AKI (relative risk [RR] 2.6, 95\%CI 2.0-3.3) compared with those not in the study. cohort at 16 hospitals(Karkouti et al., 2015). This result is different from our research, that there is no significant relationship between CPB and AKI (p-value $>0.05$ ) 
On the one hand, the time of aortic crossclamp was significantly associated with the incidence of AKI (p-value $0.006)$, increasing the incidence of AKI by 1.007 times (95\% CI 1.002-1.011)(Parolari et al., 2012). Karim et al., found that aortic cross clamp time $>60$ minutes increased the risk of CSA-AKI by 2.84 times ( $p$-value $<0.05$ )(Karim et al., 2017). Meanwhile, Sickeler et al., found that patients with a long duration of aortic crossclamp (93.4 minutes) had greater AKI compared to 85.3 minutes without AKI( $p$ value $<0.0001$ ) (Sickeler et al., 2014).

\section{CONCLUSION}

From this study it can be concluded that eGFR is an independent risk factor for AKI.

\section{REFERENCES}

[1] Aksoy R, Adedemir T, Yilmaz E, \& et al. (2019). Is Hypoalbuminemia a Predictor for Acute Kidney Injury after Coronary Bypass Grafting in Diabetes Mellitus Patients? Braz J Cardiovasc Surg, 34(5), 565-571.

[2] Alexander, J. H., \& Smith, P. K. (2016). Coronaryartery bypass grafting. In J. A. Jarcho (Ed.), New England Journal of Medicine (Vol. 374, Issue 20, pp. 1954-1964). Massachussetts Medical Society. https://doi.org/10.1056/NEJMra1406944

[3] Antonucci E, Lippi G, Ticinesi A, \& et al. (2014). Neutrophil gelatinase-associated lipocalin (NGAL): a promising biomarker for the early diagnosis of acute kidney injury (AKI). Acta Biomed, 85(1), 289-294.

[4] Arnaoutakis GJ, Vallabhajosyula P, Bavaria JE, \& et al. (2016). The impact of deep versus moderate hypothermia on postoperative kidney function after elective aortic Hemiarch repair. Ann Thorac Surg, 102, 1313-1321.

[5] Aru W; Idrus A; Marcelus S; et al. (2013). Buku Ajar Ilmu Penyakit Dalam (5th ed.). Interna.

[6] Badin J, Boulain T, Ehrmann S, \& et al. (2011). Relation between mean arterial pressure and renal function in the early phase of shock: a prospective, explorative cohort study. Crit Care, 15, 135-155.

[7] Butterworth JF; et al. (2018). Morgan and Mikhail's Clinical Anesthesiology (5th ed.). Mc Graw Hill Companies Inc.

[8] Dunser MW, Takala J, Ulmer H, \& et al. (2009). Arterial blood pressure during early sepsis and outcome. Intensive Care Med, 35, 1225-1233.

[9] Evans RG, Ince C, Joles JA, \& et al. (2013). Haemodynamic influences on kidney oxygenation: Clinical implications of integrative physiology. Clinical and Experimental Pharmacology and Physiology, 40(2), 106-122.

[10] Farhan S, Vogel B, Baber U, \& et al. (2019). Calculated Serum Osmolality, Acute Kidney Injury, and Relationship to Mortality after Percutaneous Coronary Intervention. Cardiorenal Med, 9(3).

[11] Guyton AC; Hall JE. (2016). Buku ajar fisiologi kedokteran. EGC.

[12] Karim, H., Yunus, M., Saikia, M., Kalita, J., \& Mandal, M. (2017). Incidence and progression of cardiac surgery-associated acute kidney injury and its relationship with bypass and cross clamp time. Annals of Cardiac Anaesthesia, 20(1), 22-27. https://doi.org/10.4103/0971-9784.197823
[13] Karkouti, K., Grocott, H. P., Hall, R., Jessen, M. E., Kruger, C., Lerner, A. B., MacAdams, C., Mazer, C. D., de Medicis, É., Myles, P., Ralley, F., Rheault, M. R., Rochon, A., Slaughter, M. S., Sternlicht, A., Syed, S., \& Waters, T. (2015). Rapports entre l'anémie préopératoire, l'anémie peropératoire et la transfusion de globules rouges vus comme facteurs de risque potentiellement modifiables des lésions rénales aiguës au cours de la chirurgie cardiaque: une étude de cohorte multicentrique . Canadian Journal of Anesthesia, 62(4), 377-384.

https://doi.org/10.1007/s12630-014-0302-y

[14] Kasper; Denis L; et al. (2018). Harrison's Principles of Internal Medicine 19th Edition. McGraw-Hill Education.

[15] Kidney Disease: Improving Global Outcomes (KDIGO) CKD Work Group. (2013). KDIGO 2012 Clinical Practice Guideline for the Evaluation and Management of Chronic Kidney Disease.

[16] Koponen T, Karttunen J, Musialowicz T, \& et al. (2018). Vasoactive-inotropic score and the prediction of morbidity and mortality after cardiac surgery. British Journal of Anaesthesia, 122(4), 428-436.

[17] Kowalik MM, Lango R, Klajbor K, \& et al. (2011). Incidence- and mortality-related risk factors of acute kidney injury requiring hemofiltration treatment in patients undergoing cardiac surgery: a single-center 6year experience. J Cardiothor Vasc Anesth, 25, 619-624.

[18] Kumar ABMF, Bridget Zimmerman MP, \& Suneja MM. (2014). Obesity and post-cardiopulmonary bypassassociated acute kidney injury: a single-center retrospective analysis. J Cardiothor Vasc Anesth, 28, 551-556.

[19] Mamikonian LS, Mamo LB, Smith PB, \& et al. (2014). Cardiopulmonary bypass is associated with hemolysis and acute kidney injury in neonates, infants, and children. Pediatr Crit Care Med, 15, 111-119.

[20] Manti, P., Giannakopoulos, G., Giouroukou, E., Georgaki-Angelaki, H., Stefanidis, C., \& Mitsioni, A. (2013). Psychosocial and cognitive function in children with nephrotic syndrome: association with disease and treatment variables. BioPsychoSocial Medicine, 7(10), 1-7.

https://doi.org/10.2147/IJNRD.S167477

[21] Parolari, A., Pesce, L. L., Pacini, D., Mazzanti, V., Salis, S., Sciacovelli, C., Rossi, F., \& Alamanni, F. (2012). Risk factors for perioperative acute kidney injury after adult cardiac surgery: Role of perioperative management. Annals of Thoracic Surgery, 93(2), 584-591. https://doi.org/10.1016/j.athoracsur.2011.09.073

[22] Rewa 0, \& Bagshaw SM. (2014). Acute kidney injury-epidemiology, outcomes and economics. Nature Review Nephrology, 10, 193-207.

[23] Shin, S. R., Kim, W. H., Kim, D. J., Shin, I. W., \& Sohn, J. T. (2016). Prediction and prevention of acute kidney injury after cardiac surgery. In BioMed Research International.https://doi.org/10.1155/2016/2985148

[24] Shufang L, Jingwen Z, Qian L, \& et al. (2020). Postoperative hypoalbuminemia is an independent risk factor for acute kidney injury after cardiac surgery under cardiopulmonary bypass. Chinese Journal of Nephrology, 36(8), 618-624. 
[25] Sickeler, R., Phillips-Bute, B., Kertai, M. D., Schroder, J., Mathew, J. P., Swaminathan, M., \& Stafford-Smith, M. (2014). The risk of acute kidney injury with cooccurrence of anemia and hypotension during cardiopulmonary bypass relative to anemia alone. Annals of Thoracic Surgery, 97(3), 865-871. https://doi.org/10.1016/j.athoracsur.2013.09.060

[26] Silva, A. B. V., Cavalcante, A. M. R. Z., \& Taniguchi, F. P. (2018). Survival and risk factors among dialytic acute kidney injury patients after cardiovascular surgery. Brazilian Journal of Cardiovascular Surgery, 33(3) 277-285. https://doi.org/10.21470/1678-97412017-0184

[27] Squiers, J. J., \& Mack, M. J. (2018). Coronary artery bypass grafting-fifty years of quality initiatives since Favaloro. Annals of Cardiothoracic Surgery, 7(4), 516-520. https://doi.org/10.21037/acs.2018.05.13
[28] Vives M, Hernandez A, Parramon F, \& et al. (2019). Acute kidney injury after cardiac surgery: prevalence, impact and management challenges. Int J Nephrol Renovasc Dis, 12, 153-166.

[29] Wu, Q., Yang, H., Bo, H., Fu, M., Zhong, X., Liang, G., Xu, Y., Hu, Z., Zhang, Z., Jin, X., \& Kang, Y. (2019). Predictive role of estimated glomerular filtration rate prior to surgery in postsurgical acute kidney injury among very elderly patients: a retrospective cohort study. Renal Failure.

https://doi.org/10.1080/0886022X.2019.1662440

[30] Yi, Q., Li, K., Jian, Z., Xiao, Y. Bin, Chen, L., Zhang, Y., \& Ma, R. Y. (2016). Risk factors for acute kidney injury after cardiovascular surgery: Evidence from 2,157 cases and 49,777 controls - A meta-analysis. CardioRenal Medicine. https://doi.org/10.1159/000444094 